\title{
DÜBLIN
}

Technological University Dublin

ARROW@TU Dublin

Conference Papers

Antenna \& High Frequency Research Centre

2017

\section{On the Use of Wheeler Cap for MIMO Antennas}

\author{
Florian Reher \\ Aachen University of Technology \\ Adam Narbudowicz \\ Technological University Dublin, adam.narbudowicz@mydit.ie \\ Max Ammann \\ Technological University Dublin, max.ammann@tudublin.ie
}

See next page for additional authors

Follow this and additional works at: https://arrow.tudublin.ie/ahfrccon

Part of the Electrical and Computer Engineering Commons

\section{Recommended Citation}

Reher, F., et.al. (2017). On the use of wheeler cap for MIMO antennas. EuCap: European Conference on Antennas and Propagation, Paris, France, 19th April, pg. 2691-2694. doi:10.23919/EuCAP.2017.7928579

This Article is brought to you for free and open access by the Antenna \& High Frequency Research Centre at ARROW@TU Dublin. It has been accepted for inclusion in Conference Papers by an authorized administrator of ARROW@TU Dublin. For more information, please contact arrow.admin@tudublin.ie, aisling.coyne@tudublin.ie,gerard.connolly@tudublin.ie.

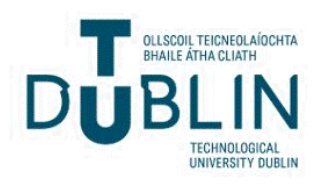




\section{Authors}

Florian Reher, Adam Narbudowicz, Max Ammann, and Dirk Heberling

This article is available at ARROW@TU Dublin: https://arrow.tudublin.ie/ahfrccon/47 


\title{
On the Use of Wheeler Cap for MIMO Antennas
}

\author{
Florian Reher ${ }^{1}$, Adam Narbudowicz ${ }^{2}$, Max J. Ammann ${ }^{2}$, Dirk Heberling ${ }^{1}$ \\ ${ }^{1}$ Institute of High Frequency Technology, RWTH Aachen University, Aachen, Germany \\ ${ }^{2}$ Antenna \& High Frequency Research Centre, Dublin Institute of Technology, Dublin, Ireland
}

\begin{abstract}
A new equivalent circuit based Wheeler Cap method to calculate MIMO efficiency is introduced. The method works without the necessity for interpolation by avoiding concur of resonating and cavity modes. The method is tested with a set of three differently correlated MIMO antennas. Results are compared with the recently proposed quality factor method for MIMO antennas and efficiency data from spherical near field measurements.
\end{abstract}

Index Terms-MIMO, efficiency, Wheeler Cap, quality factor method, equivalent circuit, correlation

\section{INTRODUCTION}

Envelope Correlation Coefficient (ECC) has become a major metric to assess the performance of MIMO antennas, i.e. to evaluate the degree of possible signal correlation due to antenna radiation patterns. This evaluation requires to measure a full-sphere radiation pattern for each port, what is time consuming and requires expensive facilities, not available to many small industries. However, an alternative technique was proposed by Hallbjorner in [1], where assuming uncorrelated losses in both antennas - one can calculate the ECC based solely on S-parameters and radiation efficiencies $\eta_{1}$ and $\eta_{2}$ :

$$
\rho_{E C C}=-\frac{S_{11} S_{12}^{*}+S_{21} S_{22}^{*}}{\sqrt{\left(1-\left|S_{11}\right|^{2}-\left|S_{21}\right|^{2}\right)\left(1-\left|S_{11}\right|^{2}-\left|S_{12}\right|^{2}\right) \eta_{\text {rad }, 1} \eta_{\text {rad }, 2}}}
$$

Measurement of antenna's efficiency is a challenging task, however ignoring the losses will yield a huge mistake in (1), as demonstrated in [1-4]. A convenient technique to overcome this difficulty could be a Wheeler Cap. Initially designed for electrically small antennas (ESA), in [5] it was proposed to extend its functionality for MIMO antennas.

The efficiency of N-port MIMO antennas is evaluated per each port $k$ and has to meet the definition for either total or radiation efficiency, viz [4]:

$$
\begin{aligned}
& \eta_{\text {total }, k}=\eta_{\text {radiation }, k} \cdot \eta_{\text {decoupling }, k} \\
& \eta_{\text {radiation }, k}=\frac{P_{\text {radiated }, k}}{P_{\text {accepted }, k}} \\
& \eta_{\text {decoupling }, k}=\frac{P_{\text {accepted }, k}}{P_{\text {in }, k}}=1-\sum_{n=1}^{N}\left|S_{n k}\right|^{2}
\end{aligned}
$$

Wheeler Cap based efficiencies are a result of at least two scattering parameter measurements. The AUT will be measured in free space and within a shielding cavity, which will reflect otherwise radiated power. However, the size of the cap as well as positioning and orientation of the antenna inside may affect the measurement [6].

Therefore, the accuracy of Wheeler Cap based radiation efficiency calculation is highly dependent on the employed post processing algorithm, which aims to filter out those effects. Several developments of Wheeler's idea consecutively enhanced its precision. Beyond the equivalent circuit employing loss and radiation resistances, two wellknown algorithms are the generalized Wheeler Cap method [7] and the Quality factor method [8, 9]. The key concept of these techniques is to compensate for inaccuracies due to resonating modes of the shielding cavity.

The quality factor method for MIMO antennas as presented in [5] is a valid post processing technique for low coupled antenna ports only. The algorithm is limited to determine efficiency from ports with $S_{21}$ of less than $-10 \mathrm{~dB}$. However, to increase the accuracy of ECC measurement, one has to take into account also strong coupling effects.

This paper proposes an equivalent circuit based post processing step for better understanding of MIMO systems. The results will be compared to Qualify Factor Method (QFM), and state-of-the-art efficiency measurement performed in anechoic chamber will be adduced.

\section{WHEELER CAP}

\section{A. Cap Eligibility}

Since equivalent circuit concept based on ohmic losses, as initially proposed by Wheeler [10], depends on accurate determination of S-parameters, one has to ensure there are no resonant cavity modes or other obscurities in the cap itself.

In its original form the method requires the shielding cavity to be located at the boundary of near and far field, so it will not restrain the antenna's radiation characteristics nor add undesired losses. Obeying this principle, the AUT will work below the cap's cut-off frequency. However, this so called radiansphere, which limits the outer boundary of the antenna's near field, in most cases cannot contain a full MIMO structure with at least two ESAs.

To avoid overlap of radiating and cavity modes the Wheeler Cap has to be examined either computationally via eigenmode solver or using mode charts - applicable for the specific shape of the cavity resonator. The AUTs operating frequency range has to meet a larger gap between excitable cavity modes (e.g. between $\mathrm{TM}_{010}$ and $\mathrm{TM}_{110}$ mode for cylindrical caps). This allows coverage of spatially coupled 
MIMO elements and scattering parameters to be measured without interacting with resonances.

\section{B. Measurement procedure}

The AUT's N-port scattering parameters will be measured in free-space and with the shielding cap to gather the needed data. Since no interpolation or smith chart circle will be applied, it is sufficient to measure over the operating frequency range only.

In case the available network analyzer does not allow Nport measurement at a stroke, other ports may be matched and measured consecutively.

Other than for the quality-factor method for MIMO antennas, no ports will be shorted during the measurements with cap.

\section{Equivalent Curcuit based Post Processing}

Equivalent circuit for the conventional measurement of single-port antenna is shown in Fig. 1. The cap short-circuits the radiation resistance $R_{\text {rad }}$, thus allowing efficiency calculation. Fig. 2 shows the equivalent circuit for the proposed MIMO enabled technique. The introduced transmission resistance $R_{\text {trans }}$ corresponds to the total impact of all other ports terminated with a matched load, which will be seen as a resistance. Please note that this approach disregards a separation of the losses from ports other than $k$. Similarly to single port implementation in $[6,10]$ the cap is expected to short-circuit the radiation resistance as shown in Fig. 2.
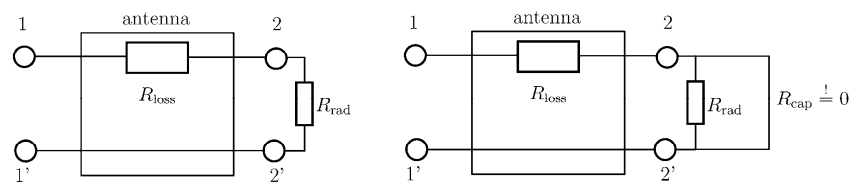

Fig. 1. Equivalent circuit diagram for single antenna
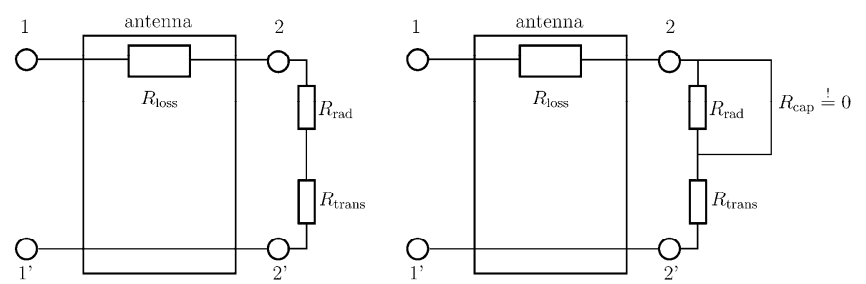

Fig. 2. Enhanced equivalent circuit diagram for MIMO antenna

To calculate the radiation efficiency for port $k$ the following formula applies, where $S_{F S}$ describes the Sparameter for the free-space radiation case and $S_{W C}$ for the measurement with Wheeler Cap:

$\eta_{\text {rad }, k}=1-\sum_{n=1}^{N}\left|S_{n k, F S}\right|^{2}-\sum_{n=1}^{N}\left|S_{n k, W C}\right|^{2}$

The total efficiency $\eta_{\text {total }}$ can be calculated by multiplying $\eta_{\text {rad }}$ with the decoupling efficiency $\eta_{\text {decouling }}$ for free-space radiation from formula (2).

\section{RESULTS}

The proposed technique was tested with Printed Inverted F Antennas (PIFA) in two MIMO constellations. The radiators (seen in Fig. 3) were etched on Rogers RT5880 substrate $\quad\left(\varepsilon_{r}=2.2, \quad \tan \delta_{\text {loss }}=0.0009 \quad\right.$ and height $h=0.508 \mathrm{~mm}$ ) and soldered perpendicular to a copper plate of dimensions $200 \times 200 \mathrm{~mm}^{2}$ serving as the ground plane [11]. This allows testing various MIMO combinations with different ECC just by re-positioning radiators. For the purpose of this study each two PIFAs were aligned in a parallel and an orthogonal position as shown in the layouts (Fig. 4).

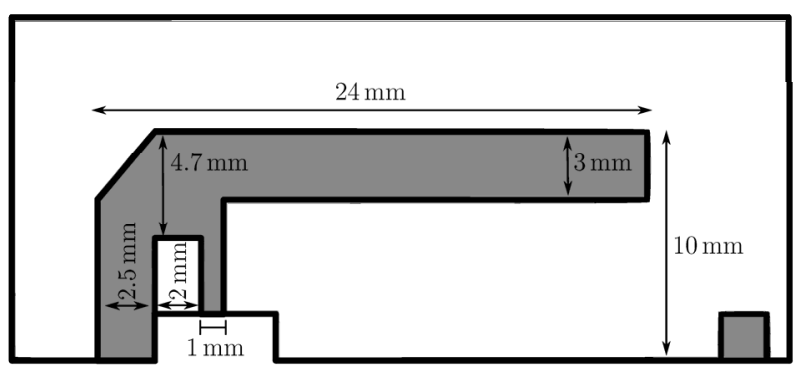

Fig. 3. PIFA radiator design for $2.5 \mathrm{GHz}$ and $150 \mathrm{MHz}$ bandwidth

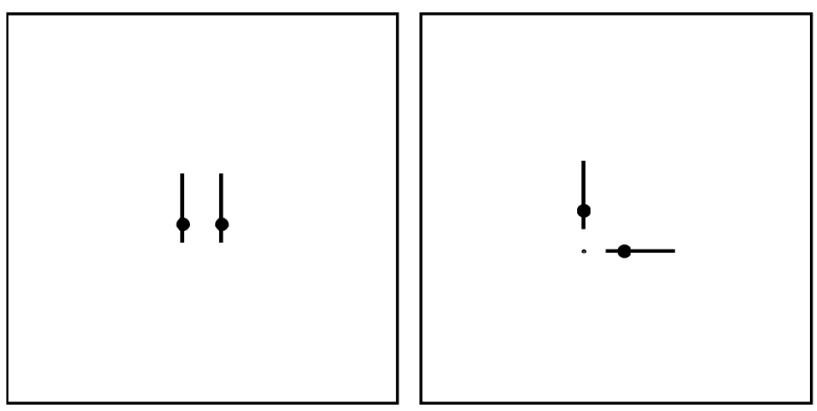

Fig. 4. MIMO arrangements on copper ground plane (topview, $20 \mathrm{~mm}$ distance between feeds respectively feed and pivot)

For ease of implementation the ground plane is to be embedded as the lower part of Wheeler Cap. The remainder of the cap is a bottom-open cylindrical cavity made from solid aluminum. It measures $121 \mathrm{~mm}$ in diameter and $54 \mathrm{~mm}$ in height.

\section{A. Efficiencies from Equivalent Circuit $W C$ method}

The implemented cylindrical Wheeler Cap exhibits a resonant $\mathrm{TM}_{010}$ mode at approximately $1.9 \mathrm{GHz}$ and $\mathrm{TM}_{110}$ as well as $\mathrm{TE}_{111}$ modes at $3 \mathrm{GHz}$. This is considered to provide sufficient separation with the investigated antennas operating frequency at $2.5 \mathrm{GHz}$.

Measured S-parameters for the orthogonal MIMO arrangement are shown as an example in Fig. 5, with parallel configuration not shown for brevity. $\mathrm{S}_{\mathrm{XX}, \mathrm{FS}}$ indicates the free space measurements, $S_{X X, W C}$ shows the Wheeler Cap measurements as described in II.B. and $\mathrm{S}_{11 \text {,QFM }}$ is measured 
with shorted-port setup to be utilized for quality factor method. The calculated total efficiencies for parallel and orthogonal configurations are shown in Figs. 6 and 7 respectively. The calculations with equivalent circuit (red solid line) were obtained with (3) and (2). It has to be emphasized, that the data rely solely on the raw S-parameter measurements and have neither been interpolated nor postprocessed in any other way. The results for parallel and orthogonal cases are in very good agreement with less than $5 \%$ absolute error, as compared to reference measurement in anechoic chamber (dotted yellow line). This demonstrates the equivalent circuit based Wheeler Cap method for MIMO can give good estimation of antenna's efficiency, while employing simplistic technique.

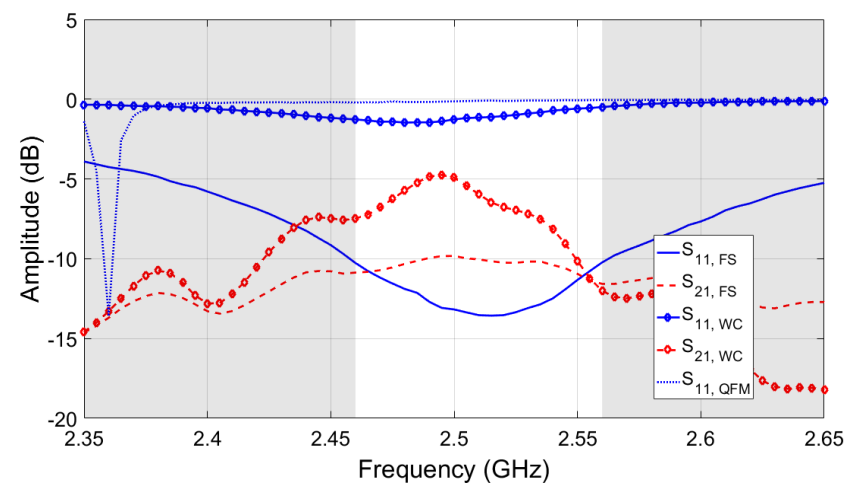

Fig. 5. Measured S-Parameters for orthogonal MIMO arrangement

\section{B. Efficiencies from Quality Factor method (QFM)}

For the QFM method antennas were measured with short-circuits placed at all ports other than $k$ to prevent coupling during measurements with Wheeler Cap [5]. A complex polynomial $\Gamma$ of degree 16 was then fitted to the measured S-parameters using least square fitting. The total efficiency has been calculated with the following equation and (2):

$$
\begin{aligned}
& \Gamma(s)=\frac{a_{n} s^{n}+a_{n-1} s^{n-1}+\ldots+a_{1} s+a_{0}}{b_{n} s^{n}+b_{n-1} s^{n-1}+\ldots+b_{1} s+b_{0}} \\
& Q=\frac{f_{\text {resonant }} \cdot|d \Gamma(s) / d f|}{1-|\Gamma(s)|^{2}} \\
& \eta_{\text {total }, Q F M, k}=\left(1-\frac{Q_{F S}}{Q_{W C}}\right) \cdot \eta_{\text {decoupling }, k, F S}
\end{aligned}
$$

Within the antenna's operating bandwidth, the QFM method performs comparably well to the equivalent circuit method. However, the parallel structure with higher coupling initially challenges the QFM and equivalent circuit at the upper part of its bandwidth. Additionally, for QFM the degree of the polynomial has to be chosen well to obtain reliable data or the calculated efficiency may have greater error - even if the S-parameter plots seem to fit at first sight. A more detailed error analysis has been published in [12].

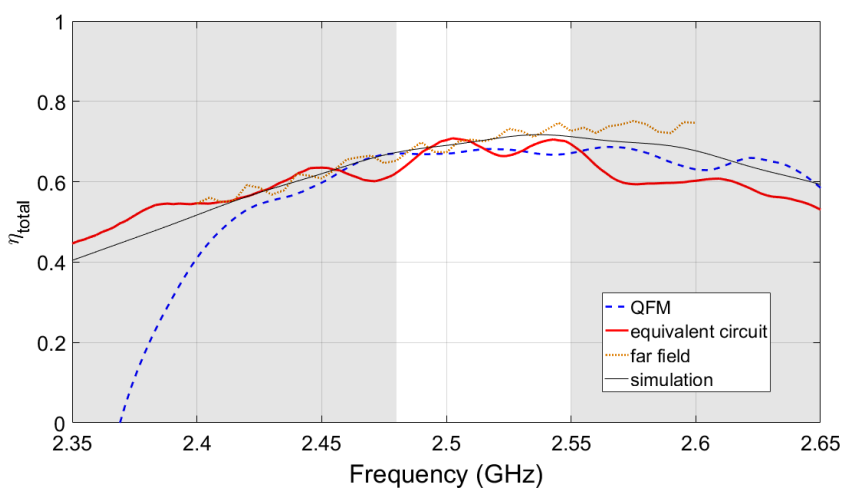

Fig. 6. Total efficiency for parallel MIMO Port 1

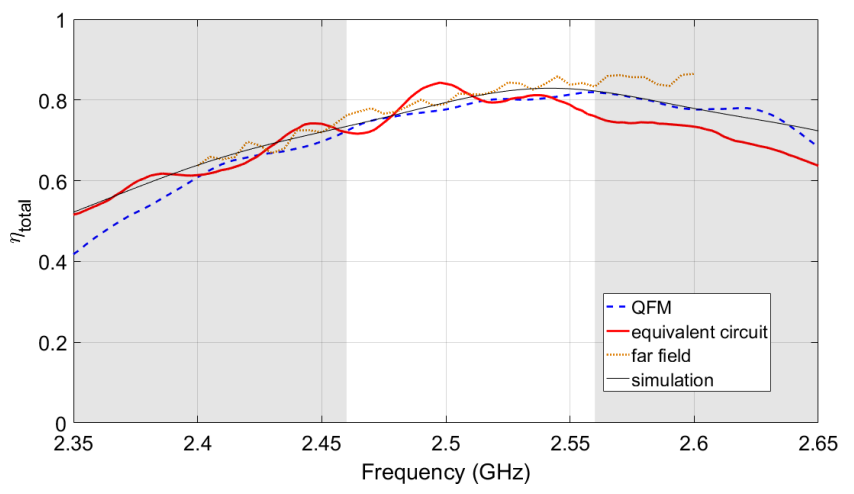

Fig. 7. Total efficiency for orthogonal MIMO Port 1

\section{VALIDATION WITH WILKINSON DIVIDER}

The previously tested MIMO antennas exhibit reasonably low correlation. To verify (3) for cases with high correlation, a single monopole antenna fed via Wilkinson divider was designed, with schematic depiction in Fig. 8. This antenna, although not practical for real life communication systems, was proposed in [2] as a testbed for MIMO correlation, as it offers a unique combination of highly correlated patterns and low $S_{2 l}$ coupling. The monopole antenna itself has very high efficiency, but due to the Wilkinson divider the total efficiency cannot exceed 0.5 . Both the equivalent circuit and the QFM were tested on simulated S-parameters (Fig. 9) (CST Microwave Studio) for antenna operating at 1.575 GHz. The efficiencies shown in Fig. 10 demonstrate some drawbacks of QFM with short-circuited ports for highly correlated and low coupled MIMO antennas. Since a short to the ground at the unused port reflects the amount of energy coupled from port 1 to 2 , the power reflected at port 1 during the QFM cap measurement is very high and misleads the algorithm from (4). Even though this is a highly theoretical examination, which would occur seldom in the field of MIMO design, it demonstrates the need for further advancements in this technique. Consequently, a combination of S-parameter based efficiency and ECC calculation with (1) is not yet advisable, since both algorithms depend on each other: high correlation might overestimate efficiency, while in turn overestimated efficiency may lead to underestimated ECC when using (1). 


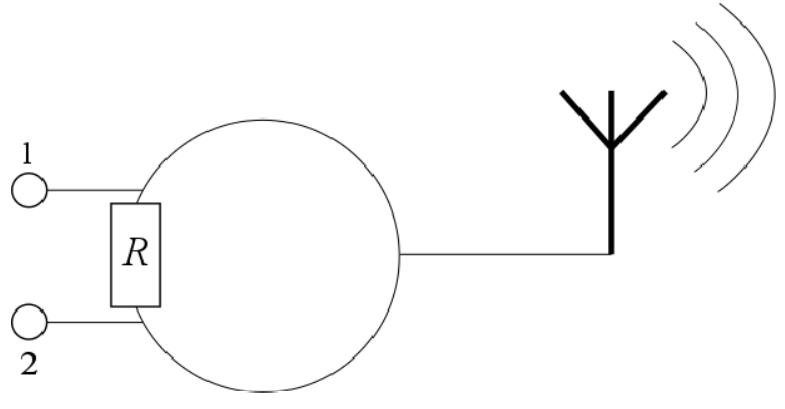

Fig. 8. Single monopole fed via Wilkinson divider

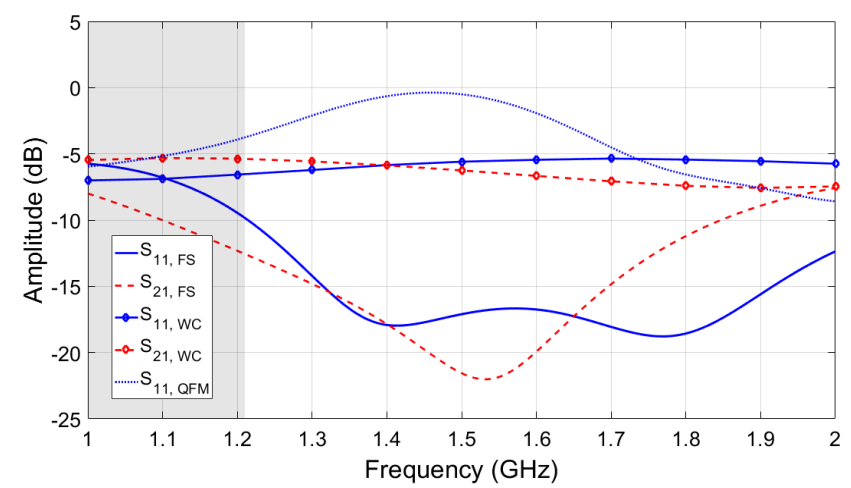

Fig. 9. Simulated S-Parameters for monopole with Wilkinson divider

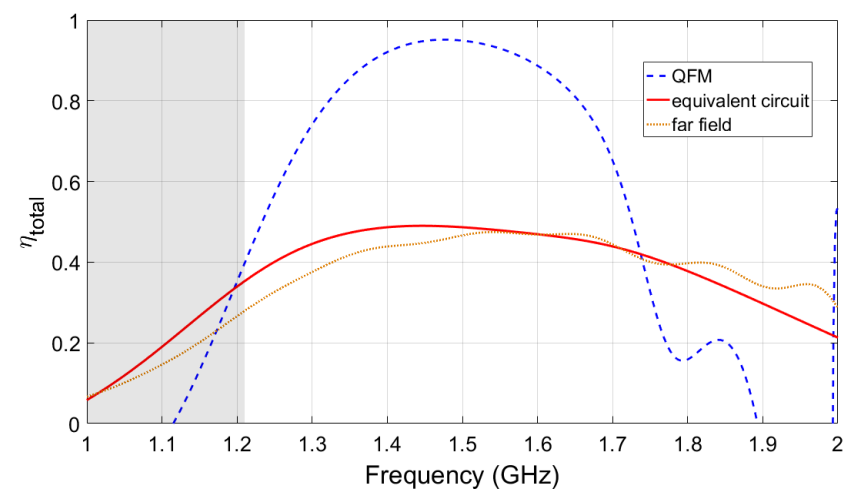

Fig. 10. Simulated total efficiency for monopole fed via Wilkinson divider

\section{CONCLUSION}

An application of Wheeler Cap method for MIMO antennas was studied. A new, simple technique using equivalent circuit model is proposed and compared to quality factor method from [5]. While the quality factor method fails to predict efficiency in cases of high coupling - as remarked in [5] -, the equivalent circuit based approach suffers from overall greater error. Nevertheless, the proposed approach seems to be valid for antennas with higher correlation. Since it is not highly dependent on polynomial fitting, its utilization comes at modest complexity.

\section{ACKNOWLEDGMENT}

This work was supported by Irish Research Council under "ELEVATE: Irish Research Council International Career Development Fellowship - co-funded by Marie Curie Actions", grant no. ELEVATEPD/2014/79.

[1] P. Hallbjorner, "The Significance of Radiation Efficiencies When Using S-Parameters to Calculate the Received Signal Correlation From Two Antennas," Antennas Wirel. Propag. Lett., vol. 4, no. 1, pp. 97-99, 2005.

[2] A. Stjernman, "Relationship between radiation pattern correlation and scattering matrix of lossless and lossy antennas," Electron. Lett., vol. 41, no. 12, p. 678, 2005.

[3] H. Li, X. Lin, B. K. Lau, and S. He, "Calculating Signal Correlation in Lossy Dipole Arrays Using Scattering Parameters and Efficiencies," in 7th European Conference on Antennas and Propagation (EuCAP), 2013: 8 - 12 April 2013, Gothenburg, Sweden, Piscataway, NJ: IEEE, 2013.

[4] A. Narbudowicz, M. J. Ammann, and D. Heberling, "Impact of lossy feed on S-parameter based envelope correlation coefficient," in 2016 10th European Conference on Antennas and Propagation (EuCAP), pp. 1-3.

[5] M. A. Moharram and A. A. Kishk, "MIMO Antennas Efficiency Measurement Using Wheeler Caps," IEEE Trans. Antennas Propagat., vol. 64, no. 3, pp. 1115-1120, 2016.

[6] M. Geissler, O. Litschke, D. Heberling, P. Waldow, and I. Wolff, “An improved method for measuring the radiation efficiency of mobile devices," in 2003 IEEE International Symposium on Antennas and Propagation: URSI North American Radio Science Meeting, pp. 743746.

[7] R. H. Johnston and J. G. McRory, "An improved small antenna radiation-efficiency measurement method," IEEE Antennas Propag. Mag., vol. 40, no. 5, pp. 40-48, 1998.

[8] R. K. Mongia, A. Ittipiboon, and M. Cuhaci, "Measurement of radiation efficiency of dielectric resonator antennas," IEEE Microw. Guid. Wave Lett., vol. 4, no. 3, pp. 80-82, 1994.

[9] M. A. Moharram and A. A. Kishk, "Modified Wheeler cap method for measurement of antenna efficiency," in 2014 XXXIth URSI General Assembly and Scientific Symposium (URSI GASS), pp. 1-4.

[10] H. Wheeler, "The Radiansphere around a Small Antenna," Proc. IRE, vol. 47, no. 8, pp. 1325-1331, 1959.

[11] F. Reher, "Study of Wheeler Cap method for multiport MIMO antennas," B.Sc. thesis, Institute of High Frequency Technology, RWTH Aachen University, Aachen, Germany, 2016.

[12] M. A. Moharram and A. A. Kishk, "Unrestricted Wideband Prediction for Antenna Radiation Efficiency Using Wheeler Caps," IEEE Trans. Antennas Propagat., vol. 64, no. 7, pp. 2869-2883, 2016. 